\title{
Competencias y actitudes para el uso de las TIC de los estudiantes del grado de maestro de Galicia
}

Competences and attitudes for the use of ICT in Galician students of the degree of teaching

加利西亚师范专业本科生使用ICT (信息与通信技术) 的能力和 态度

Навыки и отношение к использованию ИКТ студентов высших педагогических учебных заведений в Галисии (Испания)

J. Carmen Fernández de la Iglesia

Universidad de Santiago de Compostela

c.delaiglesia@usc.es

https://orcid.org/0000-0002-8320-7239

M. Carmen Fernández-Morante

Universidad de Santiago de Compostela

carmen.morante@usc.es

https://orcid.org/0000-0003-4398-3361

Beatriz Cebreiro

Universidad de Santiago de Compostela

beatriz.cebreiro@usc.es

https://orcid.org/0000-0003-2064-915X

Jorge Soto-Carballo

Universidad de Santiago de Compostela hesoto@uvigo.es

https://orcid.org/0000-0002-6768-4078

Alba-Elena Martínez-Santos

Universidad de Santiago de Compostela

alba.martinez.santos@usc.es

https://orcid.org/0000-0002-9051-9185

Lorena Casal-Otero

Universidad de Santiago de Compostela

lorena.casal@usc.es

https://orcid.org/0000-0002-0906-4321

Fechas · Dates

Recibido: 2020-01-14

Aceptado: 2020-05-20

Publicado: 2020-06-31
Cómo citar este trabajo · How to Cite this Paper

Fernández, J. C., Fernández-Morante, M. C., Cebreiro, B., Soto-Carballo, J., Martínez-Santos, A. E., \& Casal-Otero, L. (2020). Competencias y actitudes para el uso de las TIC de los estudiantes del grado de maestro de Galicia.Publicaciones, 50(1),103-120.doi:10.30827/publicaciones.v50i1.11526 


\title{
Resumen
}

La competencia para el uso de las TIC es esencial en el desempeño profesional docente. Tras décadas de estudio se observa que es necesario mejorar el nivel formativo, atendiendo a los constantes cambios generados por las tecnologías y la innovación educativa. El objetivo es conocer las competencias, usos y actitudes hacia las TIC de estudiantes del grado en maestro de educación infantil y primaria, así como contrastar de qué manera la interacción entre variables explica el uso que el alumnado realiza de las tecnologías. La muestra es de 526 estudiantes de la Universidad de Santiago de Compostela. Se usan análisis descriptivos para describir la muestra y las variables analizadas, un análisis de componentes principales para comprobar cómo se agrupaban los ítems de las escalas y análisis no paramétricos para contrastes estadísticos. Los participantes informan de una competencia media para el uso de las TIC con más dominio técnico que para el aprendizaje. A mayor nivel de competencia técnica, mayor uso de las TIC para el aprendizaje y para informarse. A mayor nivel de competencia en el uso de las TIC para el aprendizaje, mayor uso de las tecnologías para el aprendizaje. Las actitudes hacia las TIC son positivas y esto se relaciona con su mayor uso para el aprendizaje; siendo los hombres los que consideran tener más competencia para el uso. Se pone de manifiesto que la competencia para el uso de las TIC y las actitudes tienen influencia en el uso que se realiza de las mismas para aprender.

Palabras clave: Tecnologías de la Información y la comunicación; estudiantes grado maestro; competencias; actitudes

\begin{abstract}
Competence in the use of ICTs is a necessary element in the teacher professional performance, and decisions on the quality of initial teacher education need to be informed about whether or not it is appropriate at the present time. Throughout decades of national and international studies, it has been observed that it is necessary to improve the level of training in this field, taking into account the constants generated by technology and innovation in education. The aim was to describe the ICT competences, attitudes and uses that the students of the Degree in Teaching have and to contrast in what way the interaction between these factors allows to explain the use that the students make of the technologies. We use a sample constituted by 526 university students of the Degree in Teaching of the University of Santiago de Compostela. Descriptive analyses were used to describe the sample and the variables analyzed in the study. A principal component analysis was carried out to check how the items of the questionnaires were grouped (competences and attitudes) and non-parametric analysis were used to perform statistical tests. Participants reported an average level of digital competences, with the technical domain being greater than the domain for learning. The higher the level of technical competence, the greater the use of ICT for learning and to get information; also, the higher level of competence in the use of ICT for learning, the more they use it for this purpose. The participants showed positive attitudes towards ICT, and we also identified that the higher the score in positive attitudes, the greater the use of ICT for learning. Men reported a higher level of digital competences. This work showed that the digital competences of future teachers have influence in the use that these students make of them to learn. This aspect must be taken into account both by educational institutions of higher education and by other educational agents.
\end{abstract}

Key words: Information Technology; Student Teachers; competence; Attitudes 


\section{概要}

ICT应用能力对职业教师来说至关重要。经过数十年的研究, 我们发现有必要提高其培训 水平, 同时要关注技术和教育创新所带来的不断变化。本研究的目的是了解幼儿和小学师 范教育中学生对运用ICT的能力和态度, 以及检验变量之间是怎样相互影响来解释学生对 技术的应用状况。样本是来自圣地亚哥德孔波斯特拉大学的526名学生。研究中使用了描 述性分析用于描述样本和分析变量, 主成分分析用于检验量表上的项目如何分组, 以及非 参数分析用于统计检验。参与者称在ICT的平均运用能力上, 对其技术的掌握高于学习技 能。技术能力水平越高, I C T技术就越多地用于学习和对信息的了解。将ICT技术用于学习上 的能力越高, 在学习中使用的技术就越多。积极使用信息和通信技术的态度与其在学习中 的更多使用相关; 研究认为男性的ICT能力更高。结果表明, 使用信息通信技术的能力和态 度会对他们学习该技术的使用产生影响。

关键词: 信息通信技术; 师范专业本科学生; 能力; 态度

\section{Аннотация}

Компетентность в использовании ИКТ имеет важнейшее значение для профессиональной деятельности учителей. После десятилетий учебы было отмечено, что необходимо повышать уровень подготовки кадров с учетом постоянных изменений, вызванных технологическими и образовательными инновациями. Цель работы состоит в том, чтобы узнать о компетенции, использовании и отношении к ИКТ студентов учащихся на учителя дошкольного и младшего школьного возраста, а также узнать, как взаимодействие между переменными объясняет использование студентами технологий. В выборке учавствовало 526 студентов из Университета Сантьяго-де-Компостела. Описательный анализ используется для описания выборки и анализируемых переменных, анализ основных компонентов для проверки того, как группируются элементы в шкалах, и непараметрический анализ для статистических контрастов. Участники информируют о средней компетентности в использовании ИКТ с более высоким уровнем технического мастерства, чем в обучении. Чем выше уровень технической компетентности, тем выше использование ИКТ для обучения и получения информации. Чем выше уровень компетентности в использовании ИКТ для обучения, тем выше использование технологий для обучения. Отношение к ИКТ является положительным, и это связано с их более активным использованием для обучения, при этом мужчины считают себя более компетентными в их использовании. Выявлено, что компетентность в ИКТ и эмоциональное отношение к ним влияют на их использование для обучения.

Ключевые слова: информационные и коммуникационные технологии; учащиеся педагогических вузов; компетенции; отношение 


\section{Introducción}

En la sociedad actual, las Tecnologías de la Información y la Comunicación (TIC) se han convertido en un elemento fundamental con impacto en todos los sectores, incluyendo el educativo. Los recursos y los medios digitales se están convirtiendo en partes importantes de la práctica diaria de los maestros/as, teniendo cada vez más peso en la actuación en las aulas (Gudmundsdottir, \& Hatlevik, 2018). Por este motivo, la competencia en TIC del futuro profesorado ha sido un tema ampliamente investigado en los últimos años, entre otras razones, por considerarse que en su formación inicial es necesario adquirir dichas competencias para poder, posteriormente, integrar estas herramientas en las futuras prácticas de enseñanza (Aslan, \& Zhu, 2016).

En efecto, el análisis de la competencia en TIC de los/as futuros/as docentes es relevante por la vinculación directa con su futura competencia digital docente y, aún no siendo objeto de este trabajo el análisis de la misma, sí resulta necesario hacer alusión a este marco de referencia ya que se deben repensar las formas en las que se plantea la formación para un auténtico desarrollo competencial acorde con la Sociedad del Conocimiento (Cabero-Almenara, \& Palacios Rodríguez, 2019). En este sentido, tanto el Marco Europeo de Competencia Digital del Profesorado "DigCompEdu" publicado en el año 2017 (Redecker, \& Punie, 2017) como el "Marco Común de Competencia Digital Docente" definido por el INTEF (2017), constituyen herramientas fundamentales para realizar una estandarización de la competencia digital de los docentes y guiar, de este modo, la formación necesaria para los estudiantes del grado en maestro de educación infantil y primaria, ya que debemos tener presente que este hecho incide en su futura profesionalidad (Domingo-Coscolla, Carrasco, \& Sánchez, 2020).

La investigación evidencia que la competencia percibida en TIC predice significativamente la integración de las tecnologías en la futura práctica docente (Aslan, \& Zhu, 2016) y, de acuerdo con esto, centramos el objeto de este trabajo en el conocimiento de las competencias y actitudes para el uso de las TIC del alumnado del grado en maestro de educación infantil y primaria.

Tras el análisis de la literatura científica y las propuestas institucionales generadas en torno a las competencias en TIC del futuro profesorado, se identifican temas de estudio e intereses recurrentes en los últimos años, entre otros: la disponibilidad o el acceso a la tecnología de los/as futuros/as docentes (Centeno, \& Cubo, 2013; Cózar, \& Roblizo, 2014), su competencia en TIC (Aslan, \& Zhu, 2016; Cózar, \& Roblizo, 2014; Grande-de-Prado, Cañón-Rodríguez, \& Cantón-Mayo, 2016; Gutiérrez, \& Serrano, 2016; INTEF, 2017; Prendes, Castañeda,, \& Gutiérrez-Porlán, 2010; Tondeur, Aesaert, Prestridge, \& Consuegra, 2018), el uso que este alumnado realiza de la tecnología, bien sea para comunicarse, para el ocio, el aprendizaje o para acceder a la información (López-Gil, \& Bernal, 2018; Pino, \& Soto, 2010; Prendes, Solano, Serrano, González, \& Román, 2018; Tondeur et al. , 2018), las actitudes hacia las TIC (Aslan, \& Zhu, 2016; González-Martínez, Esteve-Mon, Larraz, Espuny, \& Gisbert-Cervera, 2018; Lorenz, Endberg, \& Wilfried , 2019; Maquilón, Mirete, García, \& Hernández, 2013) o si el género influye en la competencia (Centeno, \& Cubo, 2013; Cózar, \& Roblizo, 2014; Romero-Martín, Castejón-Oliva, López-Pastor, \& Fraile-Aranda, 2017; Soon, \& Ying, 2011).

La investigación ha demostrado que prácticamente el 100\% del alumnado dispone de medios y recursos vinculados con las TIC (Centeno, \& Cubo, 2013; Cózar, \& Roblizo, 2014), por lo tanto, la dotación en tecnología entre este colectivo es universal. Todos tienen, además, acceso a Internet, en su mayoría desde casa (Cózar, \& Roblizo, 2014) 
o desde su centro de estudios, ya que la red wifi está totalmente extendida en las universidades.

En relación con el nivel de competencia del futuro profesorado, se asume que los conocimientos técnicos son la base y recorren transversalmente la competencia para el uso de las TIC (Fernández de la Iglesia, Fernández Morante, \& Cebreiro, 2018; Prendes et al., 2010). En la literatura al respecto se identifican estudios que exponen hallazgos enfrentados. Por un lado, la investigación indica que los futuros docentes informan de competencias tecnológicas elevadas (Jiménez, Sancho, \& Sánchez, 2019) y manifiestan carecer de problemas en cuanto al uso, configuración e instalación de programas periféricos (Prendes et al., 2010). Por otro lado, existen estudios que constatan que los/ as futuros/as docentes perciben que tienen competencias sólo en los aspectos más básicos (Gutiérrez, \& Serrano, 2016; López, Pozo, Morales, \& López, 2019) y que no disponen de las destrezas digitales suficientes para acometer una función futura tan importante como es la educación de las nuevas generaciones de una era digital (López et al., 2019).

Si centramos nuestra atención en la competencia en TIC relacionada con áreas concretas, puede afirmarse que el alumnado informa poseer una alta competencia para informarse, comunicarse (Prendes et al., 2010) o colaborar (López et al., 2019). No obstante, la literatura pone de manifiesto que los futuros maestros/as tienen una mayor competencia para realizar actividades sencillas, como navegar por internet, y una competencia menor para manejar la información (Grande-de-Prado et al., 2016), crear contenido, atender a la seguridad y resolver problemas (Moreno, Gabarda, \& Rodríguez, 2018).

En investigaciones sobre la relación de las competencias del alumnado de magisterio se resalta que éstas no han sido adquiridas en las instituciones educativas (Centeno, \& Cubo, 2013; Marín, \& Reche, 2012), sino que una gran mayoría afirma haber aprendido sobre TIC de forma autodidacta, entre compañeros (Agreda, Hinojo, Rodríguez, \& Alonso-García, 2016; Gisbert, Espuny, \& González, 2011) o en contextos informales (López-Gil, \& Bernal, 2018).

En cuando a la frecuencia y tipos de usos que los/as estudiantes realizan de las tecnologías, se constata que los futuros maestros/as dedican mucho tiempo a las TIC, principalmente con fines académicos, comunicativos y para colaborar en el trabajo de las diferentes asignaturas de su carrera universitaria (Gisbert et al., 2011). Entendemos, y en ello coincidimos con Gisbert, González y Esteve (2016), que es necesario que el futuro profesorado posea también competencia para el uso educativo de las tecnologías que tienen a su disposición. Sin embargo, la investigación generada informa que los/ as estudiantes de magisterio se perciben a sí mismos como más competentes para el uso de las TIC que para la toma de decisiones pedagógicas relacionadas con dicho uso (Tárraga-Mínguez, Sánz-Cervera, Pastor-Cerezuela, \& Fernández-Andrés, 2017). Dicho alumnado manifiesta no estar preparado para utilizar las herramientas digitales en el proceso de enseñanza-aprendizaje (Pino, \& Soto, 2010) y señala tener dificultades para asimilar el impacto de las TIC en la sociedad y emplearlas en el proceso educativo (Grande-de-Prado et al., 2016). En esta misma línea, un estudio de Cabezas, Casillas y Pinto (2014) informa que solo el $48 \%$ de los/as futuros/as docentes se consideran competentes para aplicar las TIC en el proceso de enseñanza-aprendizaje.

La incidencia de determinadas variables en el uso educativo de las TIC ha sido un tema abordado en numerosos estudios. Entre estas variables destacan las actitudes hacia las TIC (Centeno, \& Cubo, 2013; Farjon, Smits, \& Voogt, 2019; Huda, Yulisman, Evtia 
Nurina, Erni, \& Abdullah, 2018; González-Martínez et al., 2018) y el género (López-Pastor, Fraile-Aranda, 2017; Pino, \& Soto 2010; Romero-Martín et al., 2017; Tondeur et al., 2018). En este sentido, el conjunto de investigaciones evidencia que los futuros maestros/as obtienen una puntuación relativamente alta en las actitudes y creencias hacia la tecnología (Farjon, Smits, \& Voogt, 2019; Huda et al., 2018); considerándolas interesantes, imprescindibles y esenciales para potenciar el trabajo colaborativo, actualizar su conocimiento (Centeno, \& Cubo, 2013; González-Martínez et al., 2018) y para mejorar su aprendizaje y su futuro laboral (Pino, \& Soto, 2010). Consideramos que conocer las actitudes hacia las TIC del futuro profesorado es de vital importancia, ya que éstas actúan como predictoras de la integración de las tecnologías en las prácticas de enseñanza para profesorado en formación (Aslan, \& Zhu, 2016); destacándose el impacto positivo de la actitud hacia las TIC de los/as docentes en formación y la facilidad de uso, en su posterior competencia en TIC para la práctica educativa (Lorenz et al., 2019; Pino, \& Soto, 2010; Tondeur et al., 2018). En este sentido, se confirma que la utilización de las TIC en el proceso de enseñanza-aprendizaje está directamente relacionada con el conocimiento que poseen a nivel de usuario, las actitudes, la percepción que tengan de la utilidad y potencial pedagógico de las TIC (Pino, \& Soto, 2010). Resulta importante destacar que la investigación demuestra que las actitudes del profesorado hacia el uso de las TIC en la instrucción y la colaboración y el suficiente equipamiento en TIC son predictores significativos para fomentar la competencia de los estudiantes (Lorenz et al., 2019).

Respecto a la variable género, ha sido objeto de estudio en diferentes investigaciones. En algunas de ellas se afirma que ser hombre o mujer no afecta a la percepción de competencia en TIC (Pino, \& Soto 2010; Romero-Martín et al., 2017, Tondeur et al., 2018), sin embargo, en otras, los estudiantes varones obtuvieron puntuaciones más altas que las mujeres en todas las competencias en TIC medidas (Soon, \& Ying, 2011). Atendiendo a los usos de las TIC, Pérez y Vílchez (2012) detectaron que los varones juegan más online y las mujeres emplean más las redes sociales. Las mujeres informan de un uso del ordenador superior (Cózar, \& Roblizo, 2014), son mejores mecanógrafas y tienden a usar más aplicaciones de procesamiento de textos (Soon, \&Ying, 2011).

El presente estudio ha evidenciado que la competencia en TIC de los futuros maestros/ as ha provocado un gran interés científico y se observa una férrea línea de investigación (Rodríguez-García, Trujillo, \& Sánchez, 2019) que ha generado, como hemos visto, una abundante literatura. Investigar acerca de la competencia en TIC del futuro maestro/a es una temática recurrente en el campo de la formación en tecnología en educación, necesario para continuar avanzando hacia el progreso educativo, que debe encontrarse en un proceso incesante de mejora (Rodríguez-García, Raso Sánchez, \& Ruiz-Palmero, 2019).

En este contexto se confirma la necesidad de seguir profundizando en el estudio de la competencia en TIC de los futuros docentes. Esta investigación pretende conocer las competencias para el uso de las TIC, la frecuencia y tipo de uso y las actitudes hacia estas tecnologías en una muestra de estudiantes de magisterio de educación infantil y primaria de la Universidad de Santiago de Compostela.

Este estudio se plantea los siguientes objetivos:

- Determinar el nivel de competencias que los futuros docentes tienen para el uso de las TIC

- Analizar la frecuencia y tipos de usos que los estudiantes hacen de las TIC 
- Comprobar si los participantes tienen actitudes favorables hacia las TIC

- Analizar las relaciones entre distintas variables (género, competencias, actitudes y uso) en relación con las tecnologías

\section{Metodología}

\section{Muestra}

La muestra del estudio estuvo constituida por 526 estudiantes universitarios del grado de educación infantil y primaria de la Universidad de Santiago de Compostela (Campus de Lugo y Santiago) de un total de 1280 estudiantes (porcentaje de respuesta del 41.9\%). El estudio se ha llevado a cabo entre septiembre de 2018 y mayo de 2019 . El rango de edad de la muestra estuvo entre 17 y 26 años (M=19.63; $D T=1.91)$; siendo el $85 \%$ mujeres y manifestando un $81.6 \%$ ser autodidactas en el uso de las TIC.

La participación fue voluntaria, y todos los datos fueron procesados de manera anónima. Todos los participantes dieron su consentimiento antes de ser incluidos en la muestra.

\section{Instrumentos}

Se administró un cuestionario compuesto por los siguientes apartados:

- Datos identificativos en los que se recogía información sobre género, edad, curso, si compatibilizaban estudios con trabajo, si eran autodidactas en el manejo de las TIC y acceso a las TIC.

- Escala de actitudes hacia el uso de las TIC: 16 ítems referidos a la consideración de las TIC como apoyo para su aprendizaje o futuro profesional o como obstáculo para el desarrollo del mismo.

- Escala de competencias para el uso de las TIC: compuesta por 36 ítems referidos al grado de competencia para el uso de las TIC con relación al manejo técnico y para el aprendizaje incluyendo acceso y procesamiento de la información. Para ello, los participantes evaluaban cada uno de los ítems utilizando una escala Likert de 5 puntos (codificada de 0 a 4 ) desde ninguna competencia a muy competente.

- Cuestionario de frecuencia de uso de las TIC: compuesto por 5 ítems referidos a la frecuencia de uso de las TIC para el aprendizaje, para el ocio y para informarse.

\section{Análisis y resultados}

Se realizaron los análisis estadísticos con SPSS 20.0 para Mac (IBM inc.).

Para la descripción de la muestra y las variables del estudio se han llevado a cabo análisis descriptivos (frecuencias, porcentajes y medias); se ha contrastado la validez estructural de los ítems de las escalas de actitudes hacia las TIC y de las competencias a través de un análisis de componentes principales a partir de la matriz de correlaciones. Previamente, para comprobar la idoneidad de los ítems para dicho análisis factorial, se han realizado pruebas de esfericidad de Barlett, que evalúa la existencia de 
correlación entre los ítems de cada escala, y el índice KMO (Kaiser-Meyer-Olkim), que mide el grado de intercorrelación de las variables. El número de componentes que se extrajeron se basó en la prueba scree (Cattell, 1966). Para facilitar la interpretación de los resultados obtenidos se realizó una rotación ortogonal (varimax) de los componentes extraídos. Además, se han obtenido índices de consistencia interna o fiabilidad de las dos escalas, utilizando el coeficiente alfa de Cronbach (Cronbach, 1951).

Dado que las variables de respuesta eran ordinales y no seguían una distribución normal, se llevaron a cabo análisis no paramétricos para la realización de los contrastes estadísticos, con un umbral de significación (alfa) establecido en .05.

Finalmente, para el estudio de la relación entre las variables, se obtuvieron correlaciones bivariadas de Spearman entre las dimensiones obtenidas en la escala de actitudes hacia las TIC y en la escala de competencias para el uso de las TIC y las siguientes variables: uso de las TIC para el ocio, uso de las TIC para el aprendizaje y uso de las TIC para informarse. Además, para contrastar el efecto de la variable género sobre el nivel de competencias y las actitudes hacia las TIC de los estudiantes, se realizaron pruebas U de Mann-Whitney.

Para comprobar las propiedades psicométricas de la escala de actitudes, se realizó la prueba de esfericidad de Bartlett, que confirmó la existencia de correlación entre los ítems de la escala (c2 = 2582.194; $\mathrm{p} \leq .001$ ), y el índice KMO mostró una alta correlación entre las variables ( .861$)$. El análisis de componentes principales realizado sobre los ítems de la escala de actitudes hacia las TIC mostró dos dimensiones (Tabla 1). La primera de las dimensiones se correspondía con ítems asociados a actitudes positivas hacia el uso de las TIC, explicando un $31.55 \%$ de la varianza, mientras que la segunda dimensión se correspondía con actitudes negativas hacia las TIC (12.80\% de la varianza). El análisis de fiabilidad de la escala de actitudes hacia las TIC mostró un índice alfa de Cronbach de .815 indicando, por tanto, que la escala presentaba una alta consistencia interna.

Tabla 1

Escala de actitudes

\begin{tabular}{lll}
\hline Ítem & \multicolumn{1}{c}{ Componente } \\
\hline & 1 & 2 \\
\hline Las TIC aportan mejoras a la educación & .742 \\
Las TIC son una gran ayuda para mi desempeño académico & .738 \\
El uso de las TIC incrementa las oportunidades de aprendizaje & .711 \\
El uso de las TIC constituye un elemento motivador para el aprendizaje & .707 \\
Las TIC favorecen una participación activa en el aprendizaje & .696 \\
Mi formación puede enriquecerse gracias a las posibilidades que aportan & .686 \\
las TIC & \\
Considero que las TIC incrementan y favorecen la calidad de los procesos de & .676 \\
enseñanza-aprendizaje & .670 \\
Mi competencia en TIC es importante para mi futuro profesional &
\end{tabular}




\begin{tabular}{lcc}
\hline Ítem & \multicolumn{2}{c}{ Componente } \\
\hline & 1 & 2 \\
\hline Me gusta trabajar con TIC & .548 \\
Si no actualizo mis conocimientos en TIC quedaré desfasado & .532 \\
Las TIC favorecen el trabajo en grupo & .380 \\
Las TIC dificultan el desarrollo de destrezas intelectuales básicas & .695 \\
Mi formación académica no va a mejorar por el uso de las TIC & .670 \\
Me siento desbordado por el exceso de información en la red & .662 \\
El uso de las TIC requiere una excesiva dedicación en relación a lo que me & .656 \\
aporta & .583 \\
$\begin{array}{l}\text { Debería reducirse la formación en TIC en mi carrera en favor de otros } \\
\text { aspectos más importantes }\end{array}$ & \\
\hline
\end{tabular}

En cuanto a la escala de competencias para el uso de las TIC, la prueba de Bartlett también mostró la existencia de correlación entre los ítems ( $c 2=2582.194 ; p \leq .001), y$ un índice KMO muy alto ( .948), lo que implica una alta correlación entre los ítems de la escala. El análisis de componentes principales mostró la existencia de dos dimensiones (Tabla 2), manejo técnico (explicando el 35.68\% de la varianza) y competencias de uso para el aprendizaje, que explicaba el $9.60 \%$ de la varianza. En este caso, se obtuvo un índice alfa de Cronbach de .942 indicando, nuevamente, una alta consistencia interna.

Tabla 2

Escala de competencias

\begin{tabular}{ll}
\hline Ítem & Componentes \\
\hline & 1
\end{tabular}

Sé organizar y gestionar las referencias de las fuentes de información .759

utilizadas a través de gestores bibliográficos (Refworks, Ednote, Procite...)

Sé obtener información de bases de datos educativas

Sé crear un entorno personal de aprendizaje a partir de las herramientas de $\quad .756$ la web social que conozco

Sé participar en espacios virtuales compartidos para contrastar información $\quad$.746

Sé aplicar estrategias de protección de la información para mis creaciones $\quad .731$ digitales (licencias de uso)

Sé utilizar marcadores sociales (p. ej. Diigo) para mi formación

Sé localizar repositorios de materiales educativos digitales para distintos $\quad .698$ niveles educativos

Sé utilizar y sincronizar mi calendario electrónico/virtual con otros usuarios 
Sé identificar las limitaciones de uso del material en red en base a las restricciones de propiedad intelectual

Sé suscribirme a fuentes de información relevantes para mi aprendizaje

Sé utilizar cursos virtuales online de calidad para mi formación

Sé colaborar con otras personas a distancia para elaborar un producto de información

Sé utilizar las TIC para constituir grupos de trabajo colaborativos

Sé utilizar herramientas de trabajo en grupo vía web: Groupware (Yahoo Groups, Kolab etc)

Sé utilizar redes sociales educativas (Edmodo, Eduredes, Diipo, Maestroteca, Educanetwork, Redalumnos...)

Sé compartir con otros usuarios mis creaciones digitales (documentos de texto, audio, vídeo, presentaciones multimedia...)

Sé utilizar plataformas virtuales (Moodle, Claroline...)

Sé utilizar herramientas para crear blogs (Blogger, Wordpress, Tumblr...)

Sé localizar fuentes de información relevantes para mi formación a través de redes sociales

Sé crear textos en formato digital (utilizando software como p.ej. Word, Writer, etc.)

Sé elaborar y editar presentaciones en formato digital (utilizando software como p. ej. Power Point, Impress, etc.

Sé editar textos en formato digital (utilizando software como p.ej. word, Writer, etc.)

Sé comunicarme por correo electrónico

Sé utilizar navegadores para buscar información en Internet

Sé utilizar redes sociales (p.ej. Facebook, Linkedin, Twitter, Pinterest, Instagram, Paper.li, Scoop.it...)

Sé conectar ordenador y periféricos (impresora, scanner, webcam, etc.)

Sé utilizar herramientas de comunicación síncrona via web (Chat, servicios de mensajería instantánea -tipo Messenger, Skype, otras herramientas de videoconferencia/audioconferencia, webminars, etc.)

Sé crear y editar imágenes digitales (utilizando software como p.ej. Paint NET, Photoshop, Picsizer, etc.)

Sé utilizar herramientas web para compartir/ y publicar recursos en línea (utilizando servicios como p.ej., Youtube, Flickrt, Kodak Gallery, Slideshare, Autor stream, Google docs, Scribd, etc)

Sé diseñar y gestionar Hojas de cálculo (utilizando software como p.ej. Excel, Calc, etc.) 
Sé administrar los recursos de un ordenador a través de un Sistema

.508

Operativo (p.ej. Windows, Linux, Mac, etc.)

Sé utilizar herramientas de comunicación asíncrona vía web (foros, listas de distribución, webmail, etc)

Sé crear presentaciones multimedia para enriquecer mi trabajo académico

Sé crear y editar video digital (utilizando software como p.ej. Windows Movie Maker, etc.)

Sé utilizar y gestionar software de seguridad (Antivirus, antiespias, optimizadores del sistema operativo, etc.)

Sé instalar Software

Los/as estudiantes del grado en maestro de educación infantil y primaria consideran que su formación para el uso de las TIC en su vida académica es muy escasa sobre todo en la formación previa a la universitaria, indicando el $55.5 \%$ que es nula o poca $(\mathrm{M}=1.60 ; \mathrm{DT}=.812)$. Asimismo, en el ámbito universitario el $26.9 \%$ del alumnado opina que es poca y solamente el $30.3 \%$ considera que la oferta formativa en TIC es bastante o mucha ( $\mathrm{M}=2.10 ; \mathrm{DT}=.853)$.

Dichos estudiantes han mostrado puntuaciones altas en la dimensión actitudes positivas hacia las TIC ( $M=3.20 ; \mathrm{DT}=.56)$ y puntuaciones inferiores en la dimensión actitudes negativas ( $M=1.50 ; D T=.74)$. En la escala de competencias, los/as participantes han manifestado valores medios en ambas dimensiones, siendo la media para competencias en manejo técnico de 2.49 (DT= .65) y en competencias de uso de las TIC para el aprendizaje de 2.04 (DT= .79).

Respecto a la relación entre las competencias para el uso de las TIC y el tipo de uso que hace el alumnado de las mismas, las correlaciones bivariadas mostraron que a mayor nivel de competencias técnicas se observa un mayor uso de las TIC para el aprendizaje (Rho de Spearman=.275; $p<.001$ ), para el ocio (Rho de Spearman= .198; $p<.001$ ) y para informarse (Rho de Spearman $=.258 ; p<.001)$. Estas pruebas también mostraron correlaciones significativas entre el nivel de competencias del uso de las TIC para el aprendizaje y el uso de éstas para el aprendizaje (Rho de Spearman=.337; $p<.001$ ), para el ocio (Rho de Spearman= .157; $p<.001$ ) y para informarse (Rho de Spearman= $.230 ; p<.001)$.

Las actitudes positivas hacia las TIC manifestadas por los/as participantes mostraron igualmente correlaciones significativas con el tipo de uso que hicieron de estas. Así, una mayor puntuación en actitudes positivas se correspondió con un mayor uso de las TIC para el aprendizaje (Rho de Spearman=.329; $p<.001$ ), para el ocio (Rho de Spearman= .158; $p<.001$ ) y para informarse (Rho de Spearman=.215; $p<.001)$.

Sin embargo, no se han obtenido correlaciones significativas entre actitudes negativas y el uso que el alumnado hace de las TIC.

Las pruebas $U$ de Mann-Whitney mostraron diferencias significativas en función de la variable género en competencias de uso de las TIC para el aprendizaje, siendo los hombres los que consideraban que tenían mayores competencias $(U=14464.500$, $p$ 
$=.010)$. Realizadas las pruebas para los distintos ítems de la escala de competencias (Tabla 3) se observó que se obtienen diferencias significativas en 6 de los ítems evaluados, dado que son los hombres los que informan de mayor competencia para localizar fuentes de información relevantes a través de redes sociales, participar en espacios virtuales compartidos para contrastar información, suscribirse a fuentes de información relevantes, organizar y gestionar las referencias a través de gestores bibliográficos, identificar las limitaciones de uso del material en red en base a las restricciones de propiedad intelectual y crear entornos personales de aprendizaje.

Tabla 3

Descriptivos y contrastes estadísticos por género de ítems con diferencias en competencias de uso de las TIC

\begin{tabular}{|c|c|c|c|c|c|c|}
\hline & \multicolumn{4}{|c|}{ Género } & \multicolumn{2}{|c|}{ Análisis estadídticos } \\
\hline & \multicolumn{2}{|l|}{ Mujer } & \multicolumn{2}{|c|}{ Hombre } & \multirow[b]{2}{*}{ U } & \multirow[b]{2}{*}{$\mathrm{p}$} \\
\hline & $\bar{x}$ & $\mathrm{M}_{\mathrm{e}}$ & $\overline{\mathrm{x}}$ & $\mathrm{M}_{\mathrm{e}}$ & & \\
\hline $\begin{array}{l}\text { Sé localizar fuentes de } \\
\text { información relevantes para } \\
\text { mi formación a través de redes } \\
\text { sociales }\end{array}$ & 2.75 & 3.00 & 3.00 & 3.00 & 14146.50 & .005 \\
\hline $\begin{array}{l}\text { Sé suscribirme a fuentes de } \\
\text { información relevantes para mi } \\
\text { aprendizaje }\end{array}$ & 2.09 & 2.00 & 2.35 & 2.00 & 15259.50 & .046 \\
\hline $\begin{array}{l}\text { Sé participar en espacios virtuales } \\
\text { compartidos para contrastar } \\
\text { información }\end{array}$ & 1.91 & 2.00 & 2.27 & 2.00 & 14347.00 & .007 \\
\hline $\begin{array}{l}\text { Se organizar y gestionar las } \\
\text { referencias de las fuentes de } \\
\text { información utilizadas a través de } \\
\text { gestores bibliográficos (Refworks, } \\
\text { Ednote, Procite...) }\end{array}$ & 1.47 & 1.00 & 1.75 & 2.00 & 15081.00 & .032 \\
\hline $\begin{array}{l}\text { Sé identificar las limitaciones de } \\
\text { uso del material en red en base } \\
\text { a las restricciones de propiedad } \\
\text { intelectual }\end{array}$ & 1.82 & 2.00 & 2.18 & 2.00 & 14672.00 & .014 \\
\hline $\begin{array}{l}\text { Sé crear un entorno personal } \\
\text { de aprendizaje a partir de las } \\
\text { herramientas de la web social que } \\
\text { conozco }\end{array}$ & 1.89 & 2.00 & 2.05 & 2.00 & 14480.50 & .009 \\
\hline
\end{tabular}

\section{Discusión y conclusiones}

Los objetivos de este trabajo fueron conocer las competencias, usos y actitudes que los/as estudiantes del grado de educación infantil y primaria tienen de las TIC, así 
como contrastar de qué manera la interacción entre estos factores permitía explicar el uso que el alumnado realiza de las tecnologías.

En primer lugar, según los datos recogidos en nuestra investigación, los/as estudiantes del grado de educación infantil y primaria son en su mayoría autodidactas en el aprendizaje de las tecnologías. Este dato si bien no es inesperado, pues se ha recogido en la literatura previa (Agreda et al., 2016; Centeno, \& Cubo, 2013; Gisbert et al., 2011), choca con la consideración de la competencia digital como una de las competencias clave del sistema educativo español en educación secundaria y bachillerato (Orden ECD, 65/2015), por lo que podemos concluir que el sistema educativo no está siendo lo suficientemente sistemático y eficaz en la adquisición de esa competencia clave.

Los/as futuros/as docentes perciben que tienen una competencia media en TIC, siendo mayor el dominio técnico que el dominio de las TIC para el aprendizaje. Esto corrobora resultados obtenidos en investigaciones previas (Gutiérrez, \& Serrano, 2016; López et al., 2019; Tárraga-Mínguez et al., 2017) y nos permite afirmar que esta falta de competencia percibida es una cuestión importante a tener en cuenta en los planes formativos, ya que, esta situación podría limitar futuras prácticas educativas con TIC. Nuestros resultados informan de dos correlaciones interesantes; en primer lugar, se evidencia que a mayor nivel de competencia técnica, mayor uso de las TIC para el aprendizaje y para informarse y, en segundo lugar, a mayor nivel de competencia en el uso de las TIC para el aprendizaje, más uso de las TIC para aprender. Es importante, por tanto, aumentar el nivel de competencia del futuro profesorado para influir en el uso de las tecnologías para el aprendizaje. Estos hallazgos difieren de los identificados por autores como Romero y Minelli (2011) que informan que el hecho de poseer amplias habilidades en el uso de las TIC no implica transferencia en el ámbito académico, o Pino y Soto (2010), que también afirman que, aunque el alumnado posea las destrezas mínimas necesarias para el uso de las TIC, no sabe sacar rendimiento didáctico de las mismas. Sin embargo, nuestros hallazgos están en línea con estudios más recientes que hallan una correlación estadísticamente significativa entre las horas de formación recibidas en TIC y la percepción de autoeficacia en este mismo uso educativo de las tecnologías (Tárraga-Mínguez et al., 2017).

En relación con las actitudes hemos constatado que los/as estudiantes muestran valoraciones muy positivas hacia las TIC. Dicho resultado concuerda con la literatura previa (Centeno, \& Cubo, 2013; Farjon, Smits, \& Voogt, 2019; González-Martínez et al., 2018; Pino, \& Soto, 2010). Además, la presente investigación revela que, a mayor puntuación en actitudes positivas, mayor uso de las TIC para el aprendizaje, lo que coincide con varios trabajos previos que han puesto de manifiesto esta relación (Aslan, \& Zhu, 2016; Maquilón et al., 2013; Pino, \& Soto, 2010; Tondeur et al., 2018). Se constata, por tanto, que la actitud predice el posterior uso de las TIC para aprender. Esta afirmación debe ser considerada en el diseño de la formación de los futuros maestros/as ya que, lograr en el futuro profesorado un concepto alto de autoeficacia implica que se produzcan experiencias exitosas con TIC en contextos educativos y, por tanto, un aumento en su uso (Kavanoz, Yüksel, \& Özcan, 2015).

En cuanto a la variable género, nuestros hallazgos informan que los hombres se perciben más competentes en el uso de las TIC para el aprendizaje que las mujeres; concretamente, en acciones relacionadas con el manejo y tratamiento de la información, la propiedad intelectual y la creación de entornos personales de aprendizaje. Estos resultados difieren de los hallados en estudios previos, en los que se indica que el género no afecta a la competencia en TIC (Centeno, \& Cubo, 2013; López-Pastor, \& Fraile-Aranda, 2017; Romero-Martín et al., 2017; Tondeur et al., 2018). Sin embargo, 
otros estudios sí que reconocen, en línea con el presente, diferencias de género en favor de los hombres. Así, Pino y Soto (2010) afirman que los hombres tienen una mayor competencia que las mujeres en aspectos técnicos y, Soon y Ying (2011) constatan que, aunque la diferencia no es estadísticamente significativa, los estudiantes varones todavía tienen una puntuación media ligeramente más alta en todas las competencias en TIC.

Para finalizar conviene indicar que los resultados encontrados nos permiten aportar evidencias consistentes y afirmar que la competencia en TIC del futuro profesorado tiene influencia en el uso que este alumnado realiza de las mismas para aprender. Además, tenemos que considerar que cuando se hace referencia a la competencia digital de los/as futuros/as docentes es necesario tenerlo presente en sentido amplio, recogiendo este concepto nociones relacionadas no sólo con el manejo técnico, sino con la capacidad del docente para implementar las TIC en actividades de aprendizaje y para mejorar el conocimiento y comprensión de los/as estudiantes (Spante, Sofkova, Lundin, \& Algers, 2018).

Se concluye que el alumnado del grado en maestro de educación infantil y primaria posee competencias técnicas "suficientes" para el uso de las TIC, pero inferiores para implementarlas en los procesos de enseñanza-aprendizaje (competencias didácticas y para el diseño) (Ministerio de Educación y Formación Profesional, 2019), percepción que se reafirma en las conclusiones del informe Talis 2018 respecto a la evolución de la competencia digital de los/as docentes de nuestro país. El informe pone de manifiesto una evolución en los últimos 5 años en la competencia digital del futuro profesorado, insuficiente (especialmente en secundaria) y por debajo de las medias de la UE y de la OCDE. Conviene, por tanto, que las instituciones educativas de educación superior lo tengan en cuenta dado que, como se demostró en la presente investigación, la competencia para el uso de las TIC de los futuros maestros/as en educación infantil y primaria tiene influencia en el uso que este alumnado realiza de las mismas para aprender.

\section{Referencias bibliográficas}

Agreda, M., Hinojo, M. A., Rodríguez, A. M., \& Alonso-García, S. (2016). Formación de los docentes de las Facultades de Ciencias de la Educación en TIC de la Comunidad Autónoma Valenciana. En R. Roig-Vila (Coord). Tecnología, innovación e investigación en los procesos de enseñanza-aprendizaje (pp. 1489-1498). Barcelona. Octaedro.

Aslan, A., \& Zhu, C. (2016). Investigating Variables Predicting Turkish Pre-service Teachers' Integration of ICT into Teaching Practices. British Journal of Educational Technology, 48(2), 552-570. Doi: https://doi.org/10.1111/bjet.12437

Cabero-Almenara, J., \& Palacios-Rodríguez, A. (2019). Marco Europeo de Competencia Digital Docente «DigCompEdu». Traducción y adaptación del cuestionario «DigCompEdu Check-In». EDMETIC, 9(1), 213-234. Doi: https://doi.org/10.21071/ edmetic.v9i1.12462

Cabezas, M., Casillas, S., \& Pinto, A. (2014). Percepción de los alumnos de Educación Primaria de la Universidad de Salamanca sobre su competencia digital. Edutec. Revista Electrónica De Tecnología Educativa, (48). Doi: https://doi.org/10.21556/ edutec.2014.48.156 
Cattell, R. B. (1966). The scree test for thenumber of factors. Multivariate behavioralresearch, 1(2), 245-276. Doi: 10.1207/s15327906mbr0102_10

Centeno, G., \& Cubo, S. (2013). Evaluación de la competencia digital y las actitudes hacia las TIC del alumnado universitario. Revista de Investigación Educativa, 31(2), 517-536. Doi: http://dx.doi.org/10.6018/rie.31.2.169271

Cózar, R., \& Roblizo, M. J. (2014). La competencia digital en la formación de los futuros maestros: percepciones de los alumnos de los Grados de Maestro de la Facultad de Educación de Albacete. RELATEC: Revista Latinoamericana de Tecnología Educativa, 13(2), 119-133. Doi: https://doi.org/10.17398/1695-288X.13.2.119

Cronbach, L. J. (1951). Coefficient alpha and the internal structure of tests. Psychometrika, 16(3), 297-334. Recuperado de http://128.174.199.77/psychometrika_johnson/CronbachPaper\%20(1).pdf

Domingo-Coscolla, M., Bosco, A., Carrasco Segovia, S., \& Sánchez Valero, J. A. (2020) Fomentando la competencia digitaldocente en la universidad: Percepción de estudiantes y docentes. Revista de Investigación Educativa, 38(1), 167-782. Doi: http://dx.doi.org/10.6018/rie.340551

Orden ECD/65/2015, de 21 de enero, por la que se describen las relaciones entre las competencias, los contenidos y los criterios de evaluación de la educación primaria, la educación secundaria obligatoria y el bachillerato. Boletín Oficial del Estado, 29 de enero de 2015, núm. 25, pp. 6986-7003. Recuperado de https://www.boe. es/buscar/pdf/2015/BOE-A-2015-738-consolidado.pdf

Farjon, D., Smits, A., \& Voogt, J. (2019) Technology integration of pre-service teachers explained by attitudes and beliefs, competency, access, and experience. Computers, \& Education, 130, 81-93. Doi: https://doi.org/10.1016/j.compedu.2018.11.010

Fernández de la Iglesia, C., Fernández-Morante, C., \& Cebreiro, B. (2018). Influencia de variables personales y contextuales en la integración de las TIC en el aula en Galicia. Pixel-Bit: Revista de medios y educación, 53, 79-91. Doi: 10.12795/pixelbit.2018.i53.05.

Gisbert, M. Espuny, C., \& González, J. (2011). Incotic. Una herramienta para la @utoevaluación diagnóstica de la competencia digital en la universidad. Profesorado. Revista de curriculum y formación del profesorado, 15(1), 75-90. Recuperado de https://recyt.fecyt.es/index.php/profesorado/article/view/42011

Gisbert, M., González, J., \& Esteve, F. M. (2016). Competencia digital y competencia digital docente: una panorámica sobre el estado de la cuestión. Revista Interuniversitaria de Investigación. En Tecnología Educativa, 0, 74-83. Doi: http://doi. org/10.6018/RIITE2016/257631

Grande-de-Prado, M., Cañón-Rodríguez, R., \& Cantón-Mayo, I. (2016). Competencia digital y tratamiento de la información en futuros maestros de Primaria. Educatio Siglo XXI, 34(3), 101-118. Doi: http://dx.doi.org/10.6018/j/275961

González-Martínez, J., Esteve-Mon, F. M., Larraz, V., Espuny, C., \& Gisbert-Cervera, M. (2018). INCOTIC 2.0. Una nueva herramienta para la autoevaluación de la competencia digital del alumnado universitario. Profesorado. Revista de Currículum y Formación de Profesorado, 22(4), 133-152. Doi: 10.30827/profesorado.v22i4.8401

Gutiérrez, J., \& Serrano. J. L. (2016). Evaluation and development of digital competence in future primary school teachers at the University of Murcia. Journal of New Approaches in Educational Research, 5(1), 51-56. Doi: 10.7821/naer.2016.1.152 
Gudmundsdottir, G. B., \& Hatlevik, O. E. (2018). Newly Qualified Teachers' Professional Digital Competence: Implications for Teacher Education. European Journal of Teacher Education, 41(2), 214-231. Doi: https://doi.org/10.1080/02619768.2017. 1416085

Huda, I., Yulisman, H., Evtia Nurina, C., Erni, F., \& Abdullah, D. (2018). Investigating pre-service teachers about their competencies, experiences, and attitudes towards technology integration. Journal of Physics: Conference Series, 114(1, 7). Doi :10.1088/1742-6596/1114/1/012033

INTEF (2017). Marco de Competencia Digital Docente. Recuperado de http://educalab. es/documents/10180/12809/MarcoComunCompeDigiDoceV2.pdf\%20

Jiménez, D., Sancho, P., \& Sánchez, S. (2019). Perfil del futuro docente: Nuevos retos en el marco del EEES. Contextos Educativos. Revista de Educación, 0(23), 125-139. Doi: http://dx.doi.org/10.18172/con.3471

Kavanoz, S., Yüksel, H. G., \& Özcan, E. (2015). Pre-service teachers' selfefficacy perceptions on Web Pedagogical Content Knowledge. Computers, \& Education, 85, 94-101. Doi:10.1016/j.compedu.2015.02.005

López, J., Pozo, S., Morales, M., \& López, E. (2019). Competencia digital de futuros docentes para efectuar un proceso de enseñanza y aprendizaje mediante realidad virtual. Edutec. Revista Electrónica De Tecnología Educativa, 67, 1-15. Doi: https:// doi.org/10.21556/edutec.2019.67.1327

López-Gil, M., \& Bernal, C. (2018). El perfil del profesorado en la Sociedad Red: reflexiones sobre la competencia digital de los y las estudiantes en Educación de la Universidad de Cádiz. IJERI: International Journal of Educational Research and Innovation, 11, 83-100. Recuperado de https://www.upo.es/revistas/index.php/ IJERI/article/view/3265

Lorenz, R., Endberg, M., \& Wilfried, B. (2019). Predictors of Fostering Students' Computer and Information Literacy -- Analysis Based on a Representative Sample of Secondary School Teachers in Germany. Education and Information Technologies, 24(1), 911-928. Doi: https://doi.org/10.1007/s10639-018-9809-0

Maquilón, J. J., Mirete, A. B., García, F., \& Hernández, F. (2013). Valoración de las TIC por los estudiantes universitarios y su relación con los enfoques de aprendizaje. Revista de Investigación Educativa, 31(2), 537-554. Doi: http://dx.doi.org/10.6018/ rie.31.2.151891

Marín, V., \& Reche, E. (2012). Universidad 2.0: Actitudes y aptitudes ante las TIC del alumnado de nuevo ingreso de la escuela universitaria de magisterio de la UCO. Píxel-Bit. Revista de Medios y Educación, 40, 197-211. Recuperado de https://recyt. fecyt.es/index.php/pixel/article/view/61445

Ministerio de Educación y Formación Profesional (2019). Talis 2018. Estudio internacional de la enseñanza y del aprendizaje. Informe español. Madrid: Secretaría General Técnica. Recuperado de http://www.educacionyfp.gob.es/inee/dam/ jcr:64214edb-9db9-4730-9be9-6d8cf7ed0f13/talis-2018-online-2-1.pdf

Moreno, M. D., Gabarda, V., \& Rodríguez, A. M. (2018). Alfabetización informacional y competencia digital en estudiantes de magisterio. Profesorado. Revista de Currículum y Formación de Profesorado, 22(3), 253-270. Doi: 10.30827/profesorado. v22i3.8001

Pérez, F., \& Vilchez, J. E. (2012). El uso de los videojuegos y redes sociales como predictores de la integración curricular de las TIC en estudiantes de Magisterio. 
Sphera Pública, 12, 199-215. Recuperado de http://www.redalyc.org/articulo. oa?id=29729577013

Pino, M., \& Soto, J. (2010). Identificación del dominio de competencias digitales en el alumnado del grado de magisterio. Education in the knowledge society (EKS), 11(3), 336-362. Recuperado de https://revistas.usal.es/index.php/eks/article/ view/7466

Prendes, M. P., Castañeda, L., \& Gutiérrez-Porlán, I. (2010). ICT Competences of Future Teachers. [Competencias para el uso de TIC de los futuros maestros]. Comunicar, 35, 175-182. Doi: https://doi.org/10.3916/C35-2010-03-11

Prendes, M. P., Solano, I. M., Serrano, J. L., González, V., \& Román, M. M. (2018). Entornos Personales de Aprendizaje para la comprensión y desarrollo de la Competencia Digital: análisis de los estudiantes universitarios en España. Educatio Siglo XXI, 36(2), 115-134. Doi: http://dx.doi.org/10.6018/j/333081

Redecker, C., \& Punie, Y. (2017). Digital Competence of Educators DigCompEdu. Luxembourg: Publications Office of the European Union

Rodríguez-García, A. M., Trujillo, J. M., \& Sánchez, J. (2019). Impacto de la productividad científica sobre competencia digital de los futuros docentes: aproximación bibliométrica en Scopus y Web of Science. Revista Complutense de Educación, 30(2), 623-646. Doi:10.5209/RCED.58862

Rodríguez-García, A. M., Raso, F., \& Ruiz-Palmero, J. R. (2019). Competencia digital, educación superior y formación del profesorado: un estudio de metaanálisis en la Web of Science. Pixel-Bit. Revista de Medios y Educación, 54, 65-81. Doi: https:// doi.org/10.12795/pixelbit.2019.i54.04

Romero, M., \& Minelli, J. (2011). La generación net se tambalea. Percepción del dominio de las TIC de estudiantes de magisterio. TESI, 12(3), 280-298. Recuperado de https://revistas.usal.es/index.php/eks/article/view/8492

Romero-Martín, M. R., Castejón-Oliva, F. J., López-Pastor, V. M., \& Fraile-Aranda, A. (2017). Formative Assessment, Communication Skills and ICT in Initial Teacher Training. Comunicar: Media Education Research Journal, 25(52), 73-82. Doi: https:// doi.org/10.3916/C52-2017-07

Soon, T., \& Ying, L. (2011). An Empirical Analysis of Malaysian Pre-university Students' ICT Competency Gender Differences. International Journal of Network and Mobile Technologies, 2(1). Recuperado de http://library.oum.edu.my/repository/657/1/ V2N1P2.pdf

Spante, M., Sofkova, S., Lundin, M., \& Algers, A. (2018). Digital competence and digital literacy in higher education research: Systematic review of concept use. Cogent Education, 5(1). Doi: 10.1080/2331186X.2018.1519143

Ministerio de Educación y Formación Profesional (2019). Talis, 2018. Estudio internacional de la enseñanza y del aprendizaje. Informe español. Madrid: Secretaría General Técnica

Tárraga-Mínguez, R., Sanz-Cervera, P., Pastor-Cerezuela, G., \& Fernández-Andrés, M. I. (2017). Análisis de la autoeficacia percibida en el uso de las TIC de futuros maestros y maestras de Educación Infantil y Educación Primaria. Revista Electrónica Interuniversitaria de Formación del Profesorado, 20(3), 107-116. Doi: http://dx.doi. org/10.6018/reifop.20.3.263901 
Tondeur, J., Aesaert, K., Prestridge, S., \& Consuegra, E. (2018). A multilevel analysis of what matters in the training of pre-service teacher's ICT competencies. Computers, \& Education, 122, 32-42. Doi: https://doi.org/10.1016/j.compedu.2018.03.002 